\title{
Frakturen bei Sprunggelenktotalendoprothese
}

\author{
Jan Lietz, Katja Schenk, Christoph H. Lohmann
}

\section{Zusammenfassung}

Periprothetische Frakturen können als Früh- oder als Spätkomplikation nach endoprothetischer Versorgung des oberen Sprunggelenks (OSG) auftreten. In der Literatur stellt die intraoperative Fraktur eine der häufigsten Frühkomplikationen dar, wobei die meisten Frakturen den medialen Malleolus betrafen und entsprechend osteosynthetisch versorgt wurden. Prognostisch führen diese Frakturen nur sehr selten zu einem späteren TEPVersagen. Stressfrakturen als späte Komplikation nach OSG-TEP sind selten $(0,5-2 \%)$ und werden oft im $\mathrm{Zu}$ sammenhang mit einem Malalignment, einer Instabilität, einer TEP-Lockerung oder falscher Implantatgröße beschrieben. Sie führen fast immer zur Notwendigkeit einer Revisions-OP. Neben der Osteosynthese erfordern diese Frakturen eine eingehende Ursachenanalyse und entsprechende periprothetische Eingriffe und/oder Komponentenwechsel. Durch eine gute präoperative Planung und optimale OP-Technik bei der Primärimplantation kann das Frakturrisiko, sowohl im Rahmen einer Früh- als auch einer Spätkomplikation vermindert werden.

\section{Fractures after total arthroplasty of the ankle joint}

Fractures associated with arthroplastical replacement of the ankle joint (socalled periprosthetic fractures) often occur intraoperatively or as a longterm complication. In literature the most common complications in ankle arthroplasty is the intraoperative fracture, especially the inner malleolus. These fractures are treated with standardized osteosynthetic methods. When detected and treated intraoperatively these fractures do not have a significant effect on the postoperative outcome. In contrast, stress fractures as a sign of long-term complications after ankle replacement occur rarely at 0,5 to $2 \%$. Stress fractures are commonly associated with malalignment, instability, prosthesis loosening (multiple causes) or incorrect prosthesis component size. In most cases surgical revision is required. The cause of the fracture should be analyzed precisely and treated alongside the osteosynthetic procedure. Consequently, an accurate preoperative evaluation as well as high standard surgical techniques can minimize the risks of intraoperative as well as long-term fracturing after ankle joint replacement.

\section{Einleitung}

Im Vergleich zur Hüft- und Knieendoprothetik spielt die Endoprothetik des oberen Sprunggelenks nach wie vor eine eher untergeordnete Rolle. So wurden 2012 nach Angaben des Statistischen Bundesamts in Deutschland 1234 OSGEndoprothesen implantiert. Hinsichtlich

OP-JOURNAL 2015; 31: 51-54

(c) Georg Thieme Verlag KG Stuttgart · New York DOI http://dx.doi.org/10.1055/s-0034-1383345

te jedoch deutlich verbessert werden, sodass sich die OSG-Endoprothetik, neben der OSG-Arthrodese, als eine wichtige Therapieoption zur Behandlung der fortgeschrittenen OSG-Arthrose etabliert hat [3]. Neben Design- und Verankerungsproblemen von Endoprothesen der 1. Generation spielen natürlich auch anatomische und kinematische Besonderheiten des oberen Sprunggelenks eine große Rolle. Die sehr geringen Kontaktflächen verursachen enorme Kräfte im Gelenk bzw. auf das Implantat. Zudem weist das OSG, als peripheres Gelenk, primär eine schlechtere Durchblutungssituation speziell auch der umgebenden Weichteilstrukturen auf. Häufig wird dies zusätzlich durch vorausgegangene Operationen verstärkt. Umstände, welche die Implantation der Sprunggelenkstotalendoprothese zu einer anspruchsvollen Operation mit einer langen Lernkurve machen [4]. Im folgenden Artikel soll speziell auf periprothetische Frakturen als mögliche Komplikation im Rahmen der OSG-Endoprothetik eingegangen werden.

Die erfolgreiche Implantation einer Endoprothese und deren Dauerhaftigkeit setzt die Vermeidung von Komplikationen voraus. Hier unterscheidet sich die Sprunggelenksprothese nicht von anderen endoprothetisch versorgten Gelenken. Die besonderen anatomischen Verhältnisse und die einwirkende Last bedingen eine exakte Planung und eine korrekte Implantationstechnik [5]. Hierzu sollte sich der Operateur im Vorfeld eingehende Kenntnisse über die Operationstechnik aneignen und die individuellen Gegebenheiten berücksichtigen sowie sich auf die möglichen Komplikationen während des operativen Eingriffs vorbereiten. Selbstverständlich ist der Patient in diese Überlegungen einzubeziehen und über mögliche auftretende Komplikationen aufzuklären, ebenso auch über angepasste Nachbehandlungsschemen und Auswirkungen auf das tägliche Leben. 


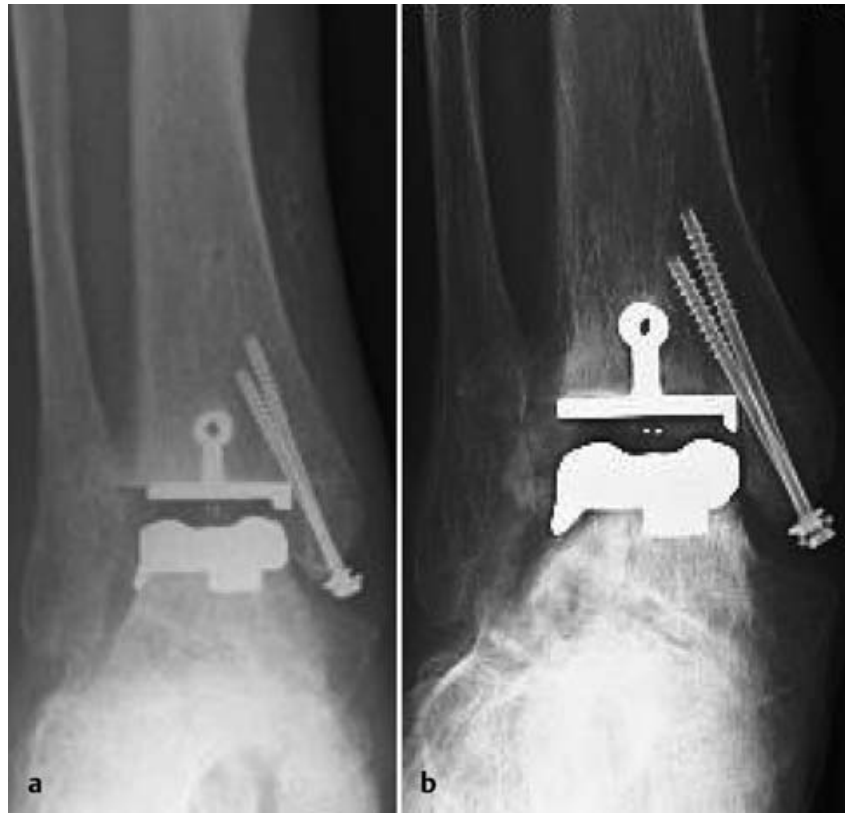

Abb. 1 a und b 72-jähriger Patient mit intraoperativer Fraktur des Malleolus medialis. a intraoperatives Bild nach Schraubenosteosynthese und OSG-TEP (Salto). b Verlaufskontrolle nach 6 Monaten.

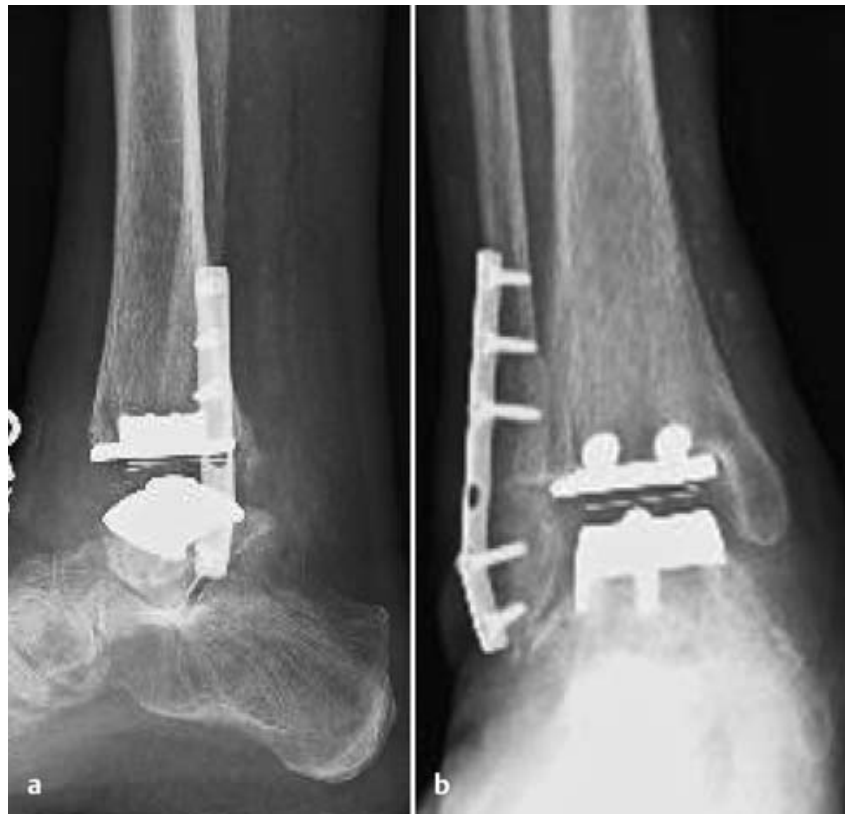

Abb. 2a und b 76-jährige Patientin 6 Monate nach OSG-TEP (Star) und osteosynthetisch versorgter Fibulafraktur.

\section{Komplikationsmöglichkeiten}

Hinsichtlich zu erwartender Komplikationen kann zwischen Früh- und Spätkomplikationen unterschieden werden.

Häufige Frühkomplikationen nach OSGTEP sind:

- intraoperative (Malleolus-)Frakturen,

- Wundheilungsprobleme,

- Fehlpositionierung der TEP und

- Infektionen.

Zu erwartende Komplikationen im späteren Verlauf sind:

- Verlust an Beweglichkeit,

- Sinterung,

- aseptische Lockerung,

- PE-Verschleiß,

- periprothetische Lysen/Zysten,

- Frakturen,

- Fehlstellung und

- Spätinfektion.

Besonders in den Anfangsjahren der OSG-Endoprothetik stellte die intraoperative Fraktur mit einer Prävalenz von bis zu 20\% ein Problem dar [6, 7]. Diese hohe Rate ist auf die geringe operative Erfahrung mit unausgereifter OP-Technik bzw. unvollkommenes Instrumentarium/Prothesendesign zurückzuführen. Auch bei Patienten mit entzündlichrheumatischer Erkrankung oder ausgeprägter Osteoporose besteht ein deutlich erhöhtes Frakturrisiko. So werden von über 29\% intraoperativen Frakturen nach OSG-TEP-Implantationen bei $\mathrm{Pa}$ - tienten mit entzündlichen Gelenkerkrankungen berichtet [8]. Mit zunehmender operativer Erfahrung und besserer OPTechnik konnte in den letzten Jahren diese spezifische Komplikationsrate deutlich verringert werden und wird heute mit ca. $8 \%$ in der Literatur angegeben [9]. In unserer Klinik traten bei über 1500 Primärimplantationen zwischen 1996 und 2013 bei 3,6\% der operierten Sprunggelenke intraoperative Frakturen und bei $0,6 \%$ postoperative Frakturen auf. Grundsätzlich können Frakturen im Rahmen der OSG-Endoprothetik in intraoperative und postoperative Frakturen eingeteilt werden.

\section{Intraoperative Frakturen}

Intraoperative Frakturen betreffen zumeist den Innen- oder Außenknöchel, seltener auch den Talus bzw. die distale Tibia. Wird während der Operation eine Fraktur vermutet, sollte eine dynamische Untersuchung unter Bildwandlerkontrolle erfolgen. Diese Frakturen werden mit den standardisierten Osteosyntheseverfahren versorgt (Abb. 1 und 2). Hierbei kommen, in Abhängigkeit vom Frakturtyp und der Lokalisation, sowohl Schrauben, Drähte, Zuggurtungsoder Plattenosteosynthesen zum Einsatz. Kleinere Fissuren im Bereich der Sägeschnitte heilen unter entsprechender postoperativer Ruhigstellung i.d.R. folgenlos aus. Besonders vorsichtig sollte die Knöchelregion bei Rheumatikern und Patienten mit Osteoporose präpa- riert werden. Hier ist gelegentlich der Einsatz von knöchelschützenden Blechen sinnvoll. Einige Autoren empfehlen auch das temporäre Einbringen von KDrähten in die Malleolen zur Vermeidung entsprechender Frakturen [7].

Neben der Sägeblattführung und -kontrolle ist auch die Positionierung und Wahl der Komponentengröße sowie der operative Zugang entscheidend. Es sollte eine ausreichende Mobilisation der Weichteile vorgenommen werden, um anatomische Landmarken genau beurteilen und Schnittlehren korrekt positionieren zu können. Nach unseren eigenen frühen Erfahrungen und Angaben in der Literatur sind die meisten Frakturen am medialen oder lateralen Malleolus iatrogen durch versehentliche Schnitte bei Verwendung einer oszillierenden Säge verursacht.

Mit steigender Implantationszahl und zunehmender operativer Erfahrung kann die Rate an Frakturen deutlich reduziert werden.

Der tibiotalare Gelenkspalt sollte beim ventralen Zugang vollständig einsehbar sein und spätere Manipulationen (Einschlagen der Implantate/Einbringen des Gleitkerns) übersichtlich zulassen. Neben der Präparation ist die korrekte Positionierung der Komponenten elementar. Wird die tibiale Komponente $\mathrm{zu}$ weit medial implantiert, begünstigt dies Frakturen am Innenknöchel. Neben der kor- 


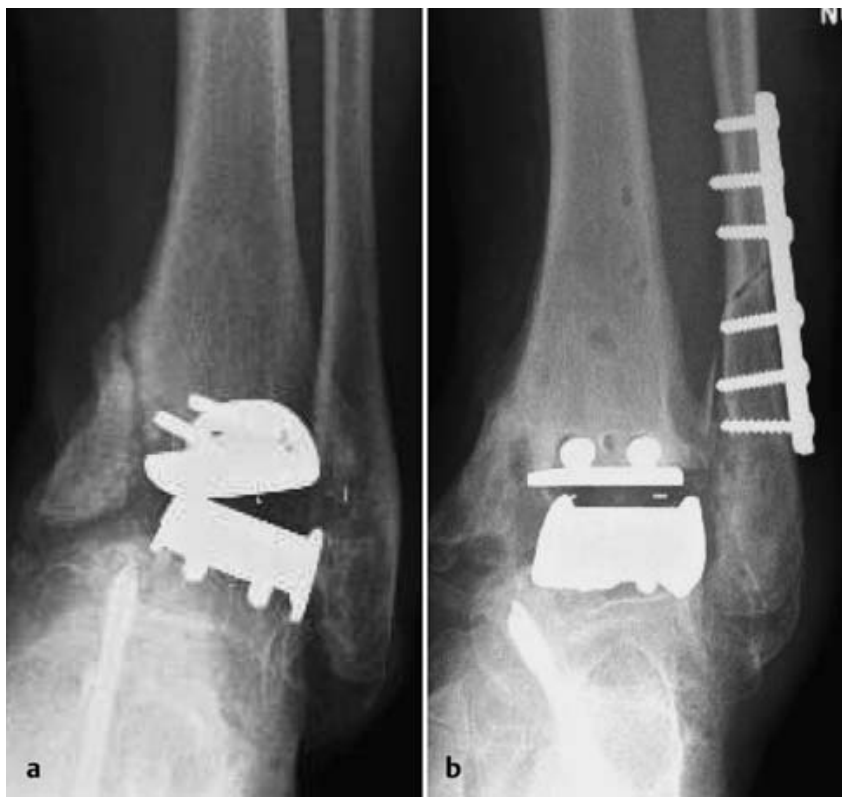

Abb. $\mathbf{a}$ a und $\mathbf{b}$ a Stressfraktur Malleolus medialis bei Varusfehlstellung nach OSG-TEP (Hintegra) und subtaler Arthrodese 6/2005. b Z.n. Revision mit OSG-TEP-Wechsel auf Star nach korrigierender Resektion und Fibula-Verkürzungsosteotomie 8/2008.

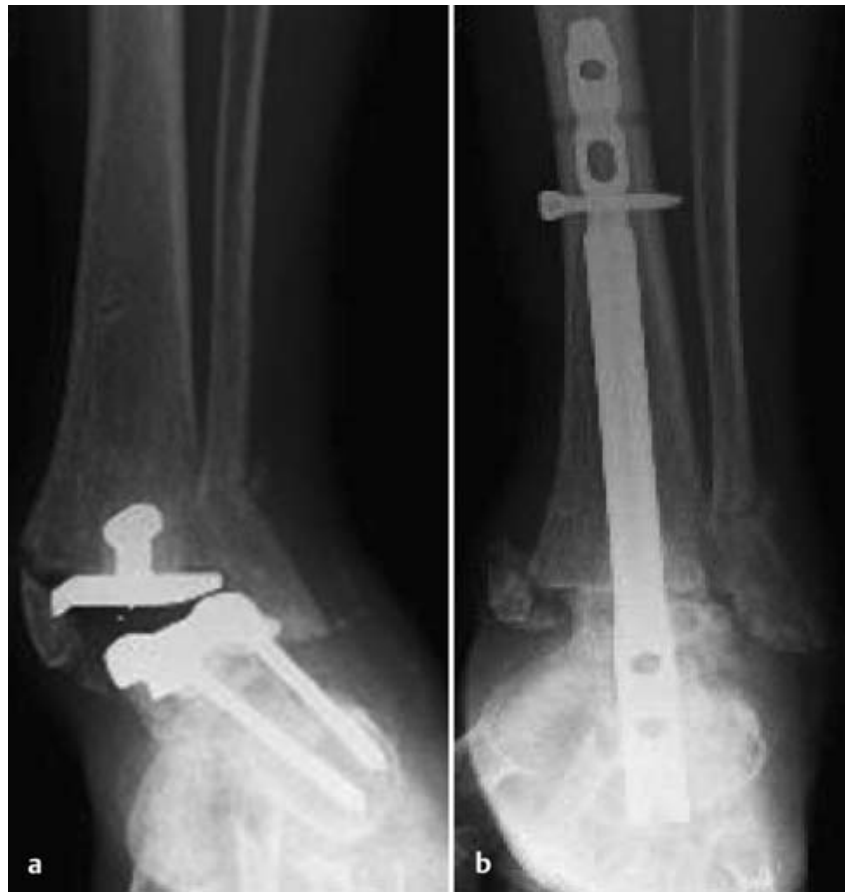

Abb. 4a und b a Bimalleoläre Stressfraktur bei 64-jähriger Patientin mit RA nach OSG-TEP (Salto) und subtalarer Arthrodese 9/2008. b Z.n. TEP-Explantation und Arthrodese mit retrogradem Verriegelungsnagel $12 / 2008$. rekten Translationsrichtung ist sowohl tibial als auch talar auf die Rotationsachse zu achten.

Bei der Implantation sollte die präoperativ avisierte Größe (basierend auf zeitnah und korrekt ausgeführten Röntgenaufnahmen) nochmals kritisch überprüft werden. Die Implantation von zu groß dimensionierten Komponenten birgt die Gefahr der Fraktur oder Schwächung der Malleolengabel [10]. Andererseits können zu kleine Implantate zu einer frühzeitigen Sinterung/Lockerung oder einem vermehrten Verschleiß durch Abrieb führen.

Das Ziel der Osteosynthese der intraoperativen periprothetischen Fraktur muss eine sichere Frakturversorgung mit Stabilität der Prothese sein, damit eine ungestörte knöcherne Integration stattfinden kann. Die Frakturen sollten dabei entsprechend den Prinzipien der AO stabilisiert werden. Nach Beendigung der Osteosynthese empfiehlt es sich, die feste Fixation der Prothese klinisch und radiologisch zu überprüfen. Postoperativ sollte auf eine entsprechende Anpassung des Nachbehandlungsschemas geachtet werden. Eine zu frühe forcierte krankengymnastische Beübung oder Belastung gefährdet u.U. den Behandlungserfolg.
Intraoperative (Malleolus-)Frakturen stellen eine häufige Komplikation bei der OSG-TEP-Implantation dar, führen jedoch bei adäquater Versorgung nur sehr selten im Verlauf zu einem Prothesenversagen.

\section{Postoperative Frakturen}

Im Vergleich zu den intraoperativen Frakturen ist dieser Frakturtyp nach Implantation einer OSG-TEP mit ca. 0,5-2\% eher selten [9]. Hinsichtlich der Ätiopathogenese werden dabei echte traumatische Frakturen (selten) und postoperative Stressfrakturen unterschieden [10]. Diese sog. Ermüdungsbrüche treten durch Fehl- bzw. Überbelastung des periprothetischen Knochens im Verlauf nach OSG-TEP-Implantation auf und sind i.d. R. Ausdruck eines schleichenden Implantatversagens [10]. Periprothetische Frakturen können zu einer TEP-Lockerung führen oder aber durch diese bedingt sein. Liegen keine Lockerungszeichen der TEP vor, so kann diese als stabil angesehen werden. Alle o.g. Kriterien haben Einfluss auf die entsprechende Therapie bzw. die Prognose der Haltbarkeit der Totalendoprothese. Nicht dislozierte Frakturen können bei stabilem Prothesensitz und korrektem Alignment auch konservativ versorgt werden.
Häufiger treten jedoch Frakturen als Stressfrakturen im Zusammenhang mit

- einer Fehlpositionierung der TEP in Varus- oder Valgusfehlstellung,

- einer zu weit medialen oder lateralen Positionierung einer oder beider Komponenten,

- einer zu groß oder klein dimensionierten Komponente oder

- einer (a)septischen TEP-Lockerung auf.

Auch Rotationsfehlstellungen, subtalare und kalkaneare Fehlstellungen oder pathologische Veränderungen im Mittelund Vorfuß können ursächlich für eine periprothetische postoperative Fraktur verantwortlich sein (Abb. 3). Es ist daher zwingend notwendig, im Hinblick auf eine notwendige Revisions-OP eine genaue und sorgfältige Ursachenanalyse durchzuführen. Prinzipiell stehen uns bei periprothetischen Frakturen verschiedene operative Optionen zur Verfügung:

- Osteosynthese der Fraktur

- Wechsel der Totalendoprothese (einoder zweizeitig)

- (korrigierende) Osteotomien (im Bereich der Fibula, Tibia, Kalkaneus oder Mittelfuß)

- Konversion in eine Arthrodese

- Bandplastiken

- Kombinationseingriffe 
Durch die zunehmende Erfahrung mit der Implantation von OSG-TEPs hat sich gezeigt, dass die korrekte Ausrichtung, das sog. Alignment, für die Langlebigkeit der Implantate ein entscheidendes Kriterium ist. Fehlpositionierungen der Komponenten und eine nicht korrekte Achsausrichtung führen zu einem gesteigerten Abrieb, aber auch zur verstärkten Anforderung an das Implantat-Knochen-Lager. Hier kann es sowohl zu überschießender Knochenbildung als auch zu Zonen reduzierten Kalksalzgehalts kommen. Der Einfluss auf die Bildung periprothetischer Zysten ist dabei noch nicht abschließend geklärt.

Insgesamt ist nicht nur die Positionierung der einzelnen Komponenten, sondern auch die Gesamtpositionierung der Prothese entscheidend. Neben den bereits angesprochenen Achsen ist auch die vorbestehende individuelle Gelenklinie für den dauerhaften Erfolg der Endoprothese entscheidend. Hier gilt es, die tibiale Komponente nicht $\mathrm{zu}$ weit proximal zu implantieren, um die knöcherne Stabilität nicht zu gefährden. Eine übermäßige tibiale Knochenresektion wird typischerweise zu einer Schwächung der medialen tibialen Säule führen und damit das Risiko für eine Tibiafraktur oder Innenknöchelfraktur erhöhen.

Neben den knöchernen Gegebenheiten ist für die Funktion der Sprunggelenkendoprothese neben der Syndesmose auch der seitliche Bandapparat entscheidend. Kommt es hier zu Insuffizienzen oder zu traumatischen Rupturen, sollten diese bei angestrebtem Erhalt der Prothese adressiert werden. Bei supramallolären periprothetischen Frakturen entscheidet meistens die Festigkeit des einliegenden Implantats über den weiteren Verlauf. Ist das Implantat noch fest knö- chern integriert, kann die Fraktur oft mit einer Plattenosteosynthese stabilisiert werden. Bei gelockerten Prothesenteilen ist oftmals nur noch die TEP-Explantation und nachfolgende Versteifung des oberen, ggf. auch des unteren Sprunggelenks, möglich. Bestehende knöcherne Defekte sollten dabei mit autologer und/ oder Fremdspongiosa aufgefüllt werden. Bei sehr ausgeprägten Defekten ist, auch im Hinblick auf die Beinlänge, das Einbringen von kortikospongiösem Knochen oder alternativ Trabekular-MetallSpacern angezeigt. Die von uns früher verwendeten Allografts in Form von z. B. halben Hüftköpfen erzielten keine zufriedenstellenden Ergebnisse.

Bei sekundär auftretenden Frakturen ist differenzialdiagnostisch auch an eine (Low-Grade-)Infektion zu denken. Hier hat sich, wie auch in der Knie- und Hüftendoprothetik, ein zweizeitiges operatives Vorgehen mit temporärer Implantation eines antibiotikahaltigen Knochenzement-Spacers bewährt. Für sehr ausgewählte Indikationen stehen heute auch Revisionsimplantate für einen TEPWechsel zur Verfügung, wobei diesbez. derzeit nur wenig Erfahrung besteht. Bei der operativen Revision sollte nach eingehender Analyse, neben der osteosynthetischen Versorgung, der Auslöser der Komplikation behoben werden. Der korrekten Ausrichtung des Rückfußes sowie dem korrekten Alignment der implantierten OSG-TEP kommt dabei eine zentrale Bedeutung zu.

Die periprothetische Stress- bzw. Ermüdungsfraktur ist eine seltene Spätkomplikation und erfordert meist eine operative Revision mit gleichzeitiger Adressierung der Ursache. Durch eine verbesserte OP-Technik kann das frühe und spätere Frakturrisiko vermindert werden.

\section{Literatur}

${ }^{1}$ Kostuj T. Erste Ergebnisse aus dem Sprunggelenksregister der D.A.F. Fuß und Sprunggelenk 10/2012; 161-165

2 Neumann HW. AE-Manual der Endoprothetik - Sprunggelenk und Fuß. Heidelberg: Springer; 2012

3 Schenk K, Lieske S, John $M$ et al. Prospective study of a cementless, mobile-bearing, third generation total ankle prosthesis. Foot Ankle Int 2011; 32: 755-763

${ }^{4}$ Gadd RJ, Barwick TW, Paling E et al. Assessment of a three-grade classification of complications in total ankle replacement. Foot Ankle Int 2014; 35: 434-437

5 Puddu G, Panni AS. Tecniche Chirurgiche in Ortopedia e Traumatologia - Total Ankle Arthroplasty Today. Roma: CIC Edizioni Internazionali; 2012

${ }^{6}$ Lieske S, Schenk K, John M et al. Die Endoprothetik des oberen Sprunggelenkes - derzeitiger Stand. OP-Journal 2007; 23: 98-103

7 McGarvey WC, Clanton TO, Lunz D. Malleolar fracture after total ankle arthroplasty: a comparison of two designs. Clin Orthop Relat Res 2004; 424: 104-110

8 Doets HC, Brand R, Nelissen RG. Total ankle arthroplasty in inflammatory joint disease with use of two mobile-bearing designs. J Bone Joint Surg Am 2006; 88: 1272-1284

${ }^{9}$ Glazebrook MA, Arsenault K, Dunbar, M. Evidence-based classification of complications in total ankle arthroplasty. Foot Ankle Int 2009; 30: 945-949

10 Manegold S, Haas NP, Tsitsilonis S et al. Periprosthetic fractures in total ankle replacement: classification system and treatment algorithm. JBone Joint Surg Am 2013; 95: 815820

\section{Jan Lietz}

Facharzt für Orthopädie und Unfallchirurgie

Dr. med. Katja Schenk

Oberärztin

Prof. Dr. med. Christoph H. Lohmann

Direktor

Orthopädische Universitätsklinik

Universität Magdeburg AöR

Leipziger Straße 44

39102 Magdeburg

jan.lietz@med.ovgu.de 